\title{
SPACE RADIO-ASTRONOMY OF SOLAR BURSTS AT \\ ALL FREQUENCIES: THE STEREO PROJECT*
}

\author{
J. L. STEINBERG and C. CAROUBALOS \\ Observatoire de Meudon, France
}

\begin{abstract}
Most observed characteristics of type III bursts are accounted for by the 'plasma hypothesis' which implies that the radio energy is radiated from the coronal altitude where the local plasma frequency is close to the observing frequency and the index of refraction vanishes. Strong focusing is to be expected in the absence of scattering effects.

Type I's are also assumed to be excited in a magnetoactive plasma near the level corresponding to the observing frequency, so that focusing effects might also be expected.

It has also been suggested that type III harmonics are radiated preferentially in the backward direction towards the Sun and that the mechanism of type I's is directive in itself.

We first show that center-to-limb histograms of occurrence cannot provide reliable information on the directivity (or focusing) of short-lived solar events as soon as it is no longer assumed that the 'polar diagram' axis is radial. The only way to provide evidence for or against directivity of solar bursts is to observe them simultaneously from the Earth and a remote space probe (STEREO-type of experiment).

We then analyse some of the information we can expect from such an experiment, for instance from the measurement of the difference in travel time of radio waves from the solar source to the earth and to the spacecraft. It is shown that the position of an 'apparent source' can be obtained from high resolution ground-based observations and space observations. Using models of the radio corona, we compute the positions of the apparent source which can be sometimes found below the photosphere, thus providing direct evidence of refractive effects in the corona and an estimation of the index of refraction at the source.

Finally, some of the problems encountered in designing STEREO-type of experiments are briefly reviewed and other examples of problems in solar physics which could be tackled using such experiments are suggested.
\end{abstract}

* Published in Astronomy und Astrophysics 9.329(1970). 